\title{
Effects of a single 30 minute walk on peripheral arterial stiffness in overweight adults
}

\author{
T. M. Kearney ${ }^{1}$, M. H. Murphy ${ }^{2}$, G. W. Davison ${ }^{2}$, M.J. O’Kane ${ }^{3}$ and A. M. Gallagher ${ }^{1}$ \\ ${ }^{1}$ Northern Ireland Centre for Food and Health, University of Ulster, Coleraine BT52 1SA, ${ }^{2}$ Ulster Sports Academy, \\ University of Ulster, Newtownabbey, BT37 OQB and ${ }^{3}$ Dept of Clinical Chemistry, Altnagelvin Hospital, Western Health \\ and Social Care Trust, Londonderry BT47 6SB, UK
}

Many of the favourable changes in cardiovascular disease (CVD) risk factors previously thought to require prolonged increases in physical activity, have now been shown in response to acute exercise ${ }^{(1)}$. Such 'last bout' effects may underlie some of the health benefits of regular physical activity. The aim of this study was to measure the effect of a single 30 minute bout of brisk walking on peripheral arterial stiffness, measured via pulse wave velocity (PWV) between the brachial and radial artery, over a $72 \mathrm{hr}$ period.

17 overweight sedentary individuals (5 males, 12 females; mean age 40 (SD 10.6) years, BMI 33.0 (SD 2.9) kg/m²) completed a 30 minute treadmill walk at $65 \%$ of their age predicted maximum heart rate. Percentage body fat (Tanita scales), BMI $\left(\mathrm{kg} / \mathrm{m}^{2}\right), \mathrm{blood}$ pressure and PWV were measured prior to the walk and PWV and blood pressure measures were then repeated at immediately post walk, and 30 mins, 60 mins, $24 \mathrm{hrs}$ and $72 \mathrm{hrs}$ post walk. PWV was measured using a sensor based device as described by McLaughlin et al. ${ }^{(2)}$. Data were analysed using a one-way ANOVA with posteriori Tukey Honestly Significant Difference (HsD) test.

There were no significant changes to the blood pressure over the 72 hours from baseline $(P>0.05)$. However the PWV increased significantly by $26 \%(P<0.004)$ immediately post-walk and declined (n.s.) by $18 \%(P>0.10)$ at 30 mins post-walk compared with preexercise reading. At 60 mins post-walk the PWV began to rise, and had returned to baseline values at 24 hr post-walk (Figure).

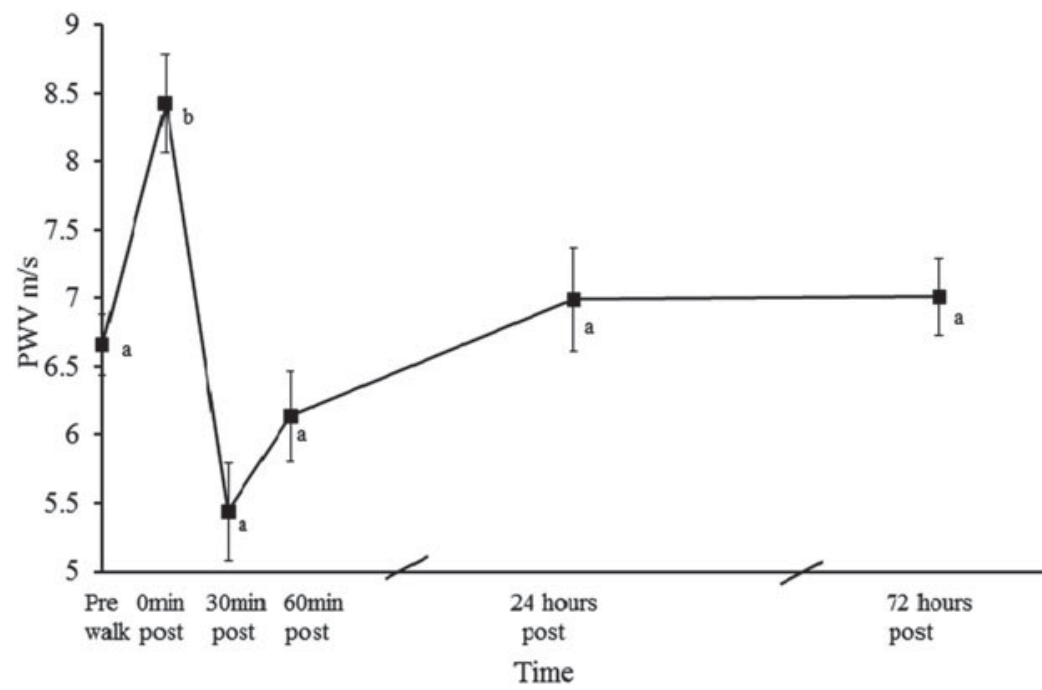

Values represent mean (with SEM) Different superscripts $(a, b)$ indicate significant differences

The increase in PWV immediately post-walk was unexpected, however since, PWV was measured within 30 sec of the participants disembarking from the treadmill; it is possible that the vasculature in the arm, where the recordings were made, was still constricted due to a redistribution of blood flow to accommodate exercising muscle. An increased cardiac output combined with vasoconstriction of the vessels in the non-exercising tissues would be required to facilitate the increased demand for oxygen in the exercising muscles of the legs ${ }^{(3)}$. Although the drop in PWV at 30 mins post walk was not significant, it is possible that had the walking been at a higher intensity, the observed drop in PWV would have been greater.

The results suggest that walking at an intensity, greater than $65 \%$ of age predicted maximum heart rate may be required to elicit a significant drop in arterial stiffness in overweight individuals.

1. Thompson et al. (2001) Med Sci Sports Exerc 33(6Suppl): S438-S445.

2. McLaughlin et al. (2003) Physiol Meas 24, 693-702.

3. MacDonald (2002) J Hum Hypertens 16(4): 225-236. 